\title{
The inflammatory effect of cardiopulmonary bypass on leukocyte extravasation in vivo
}

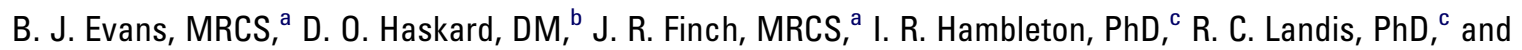 \\ K. M. Taylor, MD ${ }^{\mathrm{a}}$
}

From the British Heart Foundation, the Cardiothoracic Surgery ${ }^{\mathrm{a}}$ and Cardiovascular

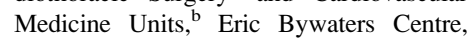
Imperial College Faculty of Medicine, Hammersmith Hospital, London, United Kingdom, and the Edmund Cohen Laboratory for Vascular Research, Chronic Disease Research Centre, ${ }^{\mathrm{C}}$ UWI, Bridgetown, Barbados.

This study was supported by grants from the British Heart Foundation (D.O.H. and K.M.T) and the Hammersmith Hospitals Trustee's Research Committee (B.J.E. and R.C.L.).

R. C. Landis reports lecture fees and Grant support from Bayer.

Received for publication March 16, 2007; revisions received Aug 22, 2007; accepted for publication Aug 30, 2007.

Address for reprints: Dr. Clive Landis, Edmund Cohen Laboratory for Vascular Research, Chronic Disease Research Centre, UWI, Jemmotts Lane, Bridgetown BB 11115, Barbados (E-mail: clive.landis@ uwichill.edu.bb).

J Thorac Cardiovasc Surg 2008;135:9991006

0022-5223/\$34.00

Copyright (C) 2008 by The American Association for Thoracic Surgery

doi:10.1016/j.jtcvs.2007.08.071
Objective: Extravascular trafficking of leukocytes into organs is thought to play a major role in the pathophysiologic mechanisms of the inflammatory response to cardiopulmonary bypass, yet leukocyte extravasation is difficult to study clinically. Here we have tested the hypothesis that leukocyte emigration into skin blisters can provide a way to monitor the inflammatory effect of cardiopulmonary bypass that allows testing of anti-inflammatory interventions (exemplified by aprotinin).

Methods: Patients undergoing primary elective coronary artery bypass grafting $(\mathrm{n}=$ 14) were randomized into 2 equal groups to receive saline infusion during cardiopulmonary bypass (control group) or high-dose aprotinin. Experimental skin blisters (in duplicate) were induced on the forearm by means of topical application of the vesicant cantharidin, and blister fluid was sampled at 5 hours postoperatively. Inflammatory leukocyte subsets in blister fluid were analyzed by means of flow cytometry by using expression of CD11b and CD62L as a phenotypic marker of activation.

Results: In the control group of patients, cardiopulmonary bypass surgery triggered a $381 \%$ increase in leukocyte extravasation into the skin compared with reference blisters carried out before surgical intervention, with neutrophil $(P=.014)$, monocyte $(P=.014)$, and eosinophil $(P=.009)$ levels all statistically significantly increased. In the aprotinin group there was no statistically significant increase during cardiopulmonary bypass surgery in any inflammatory leukocyte subset. The activation phenotype of extravascular leukocytes was not significantly altered between surgical groups.

Conclusions: This study introduces the cantharidin blister technique as a powerful new research tool for analyzing the inflammatory effect of cardiopulmonary bypass in vivo. It has provided detailed molecular insight into the extravascular leukocyte population during cardiopulmonary bypass. Although aprotinin blocked cardiopulmonary bypass-dependent extravasation of leukocytes, there was no change in their CD11b/ CD62L activation status. The cantharidin skin test thus represents a novel research tool for evaluating future anti-inflammatory interventions in cardiothoracic surgery.

I nflammation is the physiologic response to localized injury or infection and is characterized by vasodilation, increased permeability, and extravasation of inflammatory leukocytes into the affected tissue. However, a pathologic inflammatory response is triggered systemically during cardiothoracic surgery with cardiopulmonary bypass (CPB) because of contact activation of blood components by the artificial bypass circuit and can result in leukocyte infiltration and harmful cytokine secretion within susceptible organs, including the lung, kidney, and brain. ${ }^{1}$ There is increasing evidence that neurocognitive deficit after surgical intervention is linked to systemic inflammation, ${ }^{2-4}$ with a causal relationship between leukocyte infiltration and central nervous system injury demonstrated in animals. 5

Measurement of inflammatory markers in blood or urine provides little insight into the population of leukocytes that migrates extravascularly. Leukocyte extravasation in human subjects is difficult to study, with no controlled in vivo model for monitoring this process. The literature has relied mainly on animal studies of induced peritonitis 


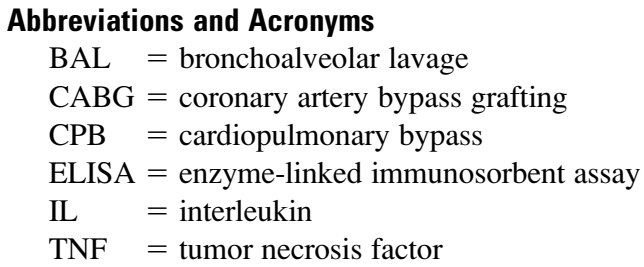

or microscopic analysis of leukocytes trafficking into animal tissues by means of intravital microscopy. However, cantharidin skin blisters have been recently described as a way to study leukocyte trafficking into an organ supplied by the systemic circulation and might thus provide a useful translational tool for monitoring the clinical inflammatory response to surgical intervention. ${ }^{7}$

Cantharidin has been in clinical use since the 1970s and is used as a topical treatment for molluscum cantagiosum and warts. It is a protein phosphatase 1 and $2 \alpha$ inhibitor. ${ }^{8}$ When applied to skin, it causes acantholysis and blister formation. ${ }^{9}$ No serious adverse reactions for topical use of cantharidin have been reported in the literature. ${ }^{10,11}$ The experimental use of cantharidin as a model to study leukocyte trafficking involves topical application of cantharidin at one seventh the clinical dose to the forearm, causing a blister (median volume, $0.5 \mathrm{~mL}$ ) to form. ${ }^{7}$ Blister fluid sampled at 24 hours exhibits the hallmarks of acute inflammation, with infiltration of inflammatory leukocytes and accretion of inflammatory cytokines, such as interleukin (IL) 8 and tumor necrosis factor (TNF) $\alpha$. We have further refined this technique to allow comprehensive analysis of the surface phenotype on emigrated leukocytes to be performed by means of flow cytometry. ${ }^{12,13}$

Aprotinin (Trasylol; Bayer Pharmaceuticals Corp, West Haven, Conn) is the only US Food and Drug Administration-licensed pharmacologic agent for limiting transfusion requirement in cardiothoracic surgery and is reported to possess clinical anti-inflammatory properties. ${ }^{14-18}$ In vitro and animal work has suggested that its anti-inflammatory properties might be mediated, at least in part, through blockade of leukocyte extravasation. ${ }^{19-22}$ In the present study we have used aprotinin as a model anti-inflammatory regimen to address the hypothesis that cantharidin skin blisters can be used to monitor anti-inflammatory intervention in coronary artery bypass grafting $(\mathrm{CABG})$ surgery with $\mathrm{CPB}$.

Our approach was to randomize patients undergoing primary elective CABG surgery with CPB into 2 groups: a control group receiving saline and an anti-inflammatory intervention group receiving full-dose aprotinin ("Hammersmith dose") during surgical intervention. Skin blisters in duplicate were sampled at 5 hours after the operation and compared between treatment groups. Further comparisons were made to reference blisters carried out before that operation, which were identical in all respects except for the absence of surgical intervention. This study design allowed us to test the hypothesis that CPB causes enhanced leukocyte extravasation into skin and that cantharidin blisters can be used to monitor anti-inflammatory intervention in cardiothoracic surgery.

\section{Materials and Methods Reagents}

Cantharidin (Cantharone) was purchased from Dormer Laboratories Inc (Rexdale, Ontario, Canada). Anti-CD62L-fluorescein isothiocyanate, anti-CD11b-R-phycoerythrin, control IgG- fluorescein isothiocyanate, and control IgG-R-phycoerythrin were purchased from BD Biosciences (Oxford, United Kingdom). Anti-CD14-phycoerythrinTexas red conjugate and control IgG-phycoerythrin-Texas red conjugate were purchased from Beckman Coulter (High Wycombe, United Kingdom).

\section{Patients}

Institutional review board approval for the study protocol was gained from the Hammersmith, Queen Charlottes, and Chelsea Research Ethics Committee. Patients referred for elective CABG were enrolled after providing informed consent, and the study was registered with www.Clinicaltrials.gov (identification number: NCT00131040). Exclusion criteria were emergency cases, combined valvular procedures or redo operations, unstable angina or myocardial infarction within 6 weeks before surgical intervention, cerebrovascular accident within 3 months preoperatively or more than $75 \%$ carotid artery obstruction (as shown by carotid Doppler scanning), serum creatinine level in excess of $177 \mu \mathrm{mol} / \mathrm{L}$, coagulopathy, or previous use of anticoagulant/antiplatelet agents within the week before surgical intervention. Patients who satisfied the exclusion criteria were randomized in a double-blind study design to receive saline infusion during bypass (group $1, \mathrm{n}=7$ ) or highdose ("Hammersmith dose") aprotinin $\left(2 \times 10^{6}\right.$ KIU loading dose, $2 \times 10^{6} \mathrm{KIU}$ in the pump prime, followed by $0.5 \times 10^{6}$ $\mathrm{KIU} / \mathrm{h} \mathrm{CPB}$; group 2, $\mathrm{n}=7$ ). CPB was performed with aortic and right atrial cannulation. The extracorporeal circuit consisted of a roller pump (Stockert Instruments, Munich, Germany), a Bard William Harvey HF-750 membrane oxygenator (C.R. Bard, Crawley, England), and polyvinyl chloride tubing (non-albumin coated). Pulsatile extracorporeal circulation was used at 2.2 to $2.4 \mathrm{~L} \cdot \mathrm{m}^{-2}$. $\min ^{-1}$ to maintain a mean blood pressure of 50 to $60 \mathrm{~mm} \mathrm{Hg}$. Moderate hypothermia of $32^{\circ} \mathrm{C}$ to $34^{\circ} \mathrm{C}$ was applied in all patients. Patients received heparin (Monoparin; CP-Pharmaceuticals, Wrexham, United Kingdom) to achieve an activated coagulation time of greater than 480 seconds (Hemochron Jr, [International Technidyne Corporation, Edison, NJ] using kaolin).

\section{Cantharidin Skin Blisters}

The cantharidin skin blister technique has been described in detail previously. ${ }^{7}$ Skin blisters in duplicate were induced by means of topical application of a $0.1 \%$ solution of Cantharone (Dormer Laboratories) in acetone to the volar aspect of the forearm according to the timeline depicted in Figure 1. Each blister was allowed to develop for 24 hours, with perioperative blisters timed for harvest at 5 hours after the initiation of CPB. Blister fluid was collected with siliconized pipette tips (Sigma Aldrich, Poole, United Kingdom) 


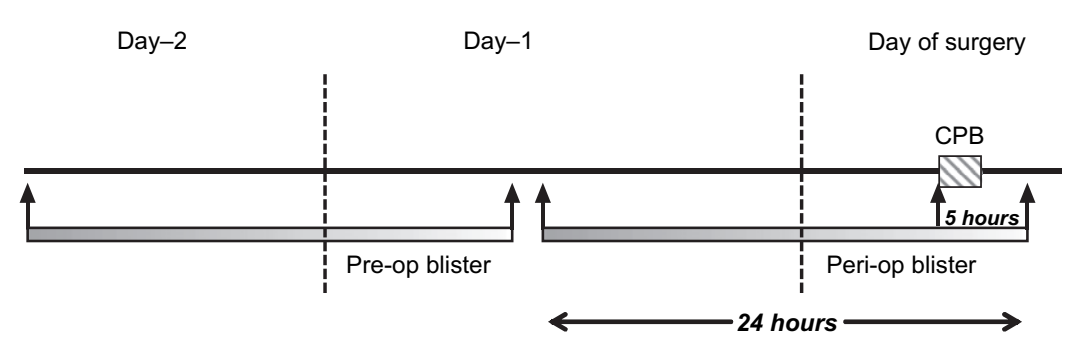

and stored on ice in siliconized microcentrifuge tubes before flow cytometric analysis. Cell counts were performed with Kimura's stain and counting in a hemocytometer. Cell-free supernatants, collected after microcentrifugation, were stored at $-70^{\circ} \mathrm{C}$ for enzymelinked immunosorbent assay (ELISA) analysis.

\section{Flow Cytometric Analysis}

$\mathrm{CD} 11 \mathrm{~b}$ and CD62L expression was monitored on gated neutrophil and monocyte populations in whole blood and blister infiltrated cells, according to the gating scheme depicted in Figure 2. Flow cytometric analysis of blister leukocytes was performed immediately after centrifugation of blister samples, with all incubation (15 minutes) and washing steps being carried out in ice-cold phosphatebuffered saline, as previously described. ${ }^{13}$ Whole-blood samples were prepared for staining by using the Immunolyse whole-blood lysing technique (Beckman Coulter, Luton, United Kingdom), as previously described. ${ }^{23}$ Primary antibody incubations were carried out in phosphate-buffered saline at room temperature for $15 \mathrm{~min}$ utes, as per the manufacturer's recommendations. Results are expressed as mean fluorescent staining intensities.

\section{ELISAs}

IL- 8 and TNF- $\alpha$ levels in blister fluid were measured in triplicate by means of capture ELISA (R\&D Systems, Abingdon, United Kingdom), according to the manufacturer's recommendations. TNF levels showed wide interpatient variability, and results were normalized for each individual by using preoperative blisters. IL-8 levels were expressed as the mean concentration (in nanograms per milliliter) \pm the standard error.

\section{Statistical Analysis}

Power studies based on a previous investigation ${ }^{24}$ of leukocyte extravasation into skin blisters in healthy donors indicated that a minimum sample size of 5 in each group would have a greater than $90 \%$ power to detect a difference in means of $7.50 \times 10^{5}$ cells per blister (the difference between a group 1 mean of $15.00 \times 10^{5}$ cells per blister and a group 2 mean of $7.50 \times 10^{5}$ cells per blister), assuming that the common standard deviation was 2.8 by using a 2-group $t$ test with a .05 2-sided significance level.

Differences in perioperative patient characteristics between treatment groups were analyzed by using distribution-free univariate tests: the Fisher exact test for 2-level categorical data, such as sex and diabetes prevalence; the Wilcoxon Mann-Whitney test for 3level categorical data, such as Canadian Cardiovascular Society class and number of grafts; and a permutation test for continuous data, such as age and blood loss.

All leukocyte components were log-transformed before analyses to improve the normality of the data. After averaging results from duplicate blisters (there were 2 blisters per person at each time point), differences between preoperative and perioperative leukocyte components were assessed by using paired $t$ tests. Treatment group differences in perioperative leukocyte components were analyzed by using unpaired $t$ tests. The average values of leukocyte components were presented as geometric means. ${ }^{25}$ Because of the small sample size, all parametric tests were verified by using an equivalent distribution-free test (the Wilcoxon matched pairs signed-rank test for a paired $t$ test and the Mann-Whitney $U$ test for an unpaired $t$ test).

Exact $P$ values are presented at all times to clarify the strength of the statistical relationships. Statistical analysis was performed with Stata 9 statistical software (Stata Corp, College Station, Tex).

\section{Results}

\section{Study Design and Group Characteristics}

The study design is outlined in Figure 1. Patients scheduled for primary elective $\mathrm{CABG}$ with $\mathrm{CPB}$ were enrolled into the study during the presurgery clinic with institutional review board approval and after providing informed consent. Patients who satisfied the exclusion criteria were randomized into control (saline) and aprotinin treatment groups. Study participants were invited to book into the presurgical ward 2 days before the operation and had reference skin blisters, in duplicate, carried out on their forearm ("pre-op blister" in Figure 1). These blisters would serve as an internal control to establish each person's baseline response to cantharidin. Perioperative skin blisters were then induced on the day before the operation and timed such that collection of blister fluid would take place postoperatively 5 hours after the initiation of CPB ("peri-op blister" in Figure 1).

Experimental cantharidin skin blisters were well tolerated by patients. No statistically significant differences in patient demographics or preoperative characteristics were noted between the aprotinin and saline treatment groups (Table 1). 


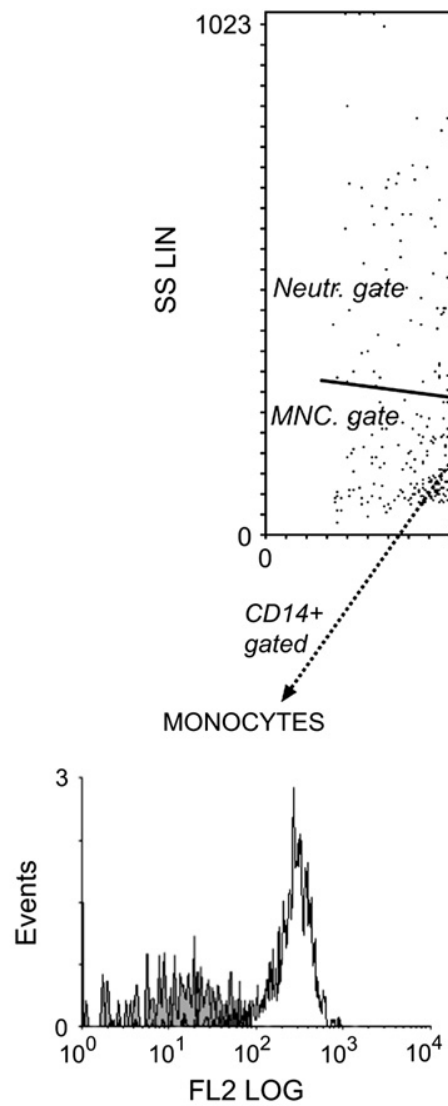

Figure 2. Flow cytometric dot plot of blister fluid illustrating the gating strategy used to discern extravascular neutrophil and monocyte subpopulations. Neutrophils were identified and gated based on their characteristic side-scatter profile. Monocytes were identified in the mononuclear cell (MNC) gate based on expression of CD14 in the fluorescence (FL) 3 channel. Representative flow histograms depict CD11b and CD62L expression perioperatively on neutrophils and monocytes present in blister fluid (solid line) versus the circulation (filled histograms). SS, side scatter; FS, forward scatter; LIN, linear.

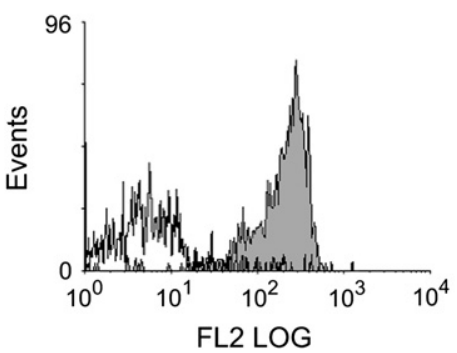

CD62L

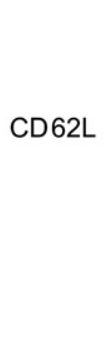

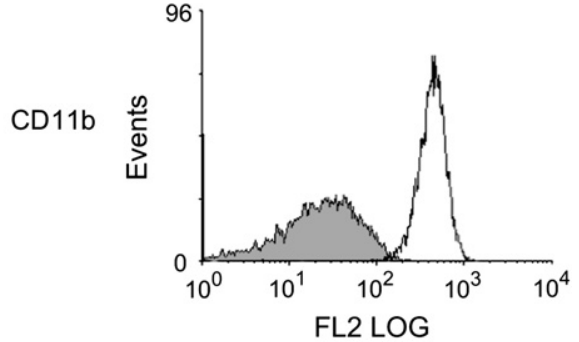

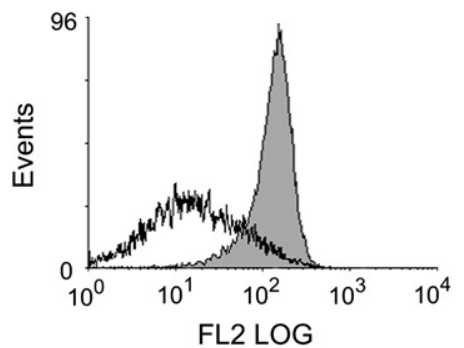

There was no statistically significant difference in the number of grafts performed, bypass time, or crossclamp time. At follow-up, there was significantly less blood loss $(P=.02)$ and red cell transfusion $(P=.01)$ in the aprotinin group. No statistically significant differences were noted for platelet transfusion, serum creatinine level, or atrial fibrillation. No serious adverse events after surgical intervention were reported in either group (Table 1).

\section{Leukocyte Extravasation into Skin Blisters}

In the saline control group leukocyte extravasation into perioperative skin blisters increased from 4.84 (95\% CI, 2.14-0.92) to 24.48 (95\% CI, 10.30-58.16) compared with reference blisters harvested preoperatively $(P=.013$, Table 2$)$, which represents a $381 \%$ increase in leukocyte emigration in- duced by surgical intervention. Inflammatory cells recovered from blisters were analyzed flow cytometrically by using the gating strategy shown in Figure 2 (upper panel). Neutrophils were resolved on the basis of their characteristic side-scatter profile, as previously described, ${ }^{13}$ and comprised $94.6 \% \pm$ $3.96 \%$ (mean \pm standard deviation) pure neutrophils $\left(\mathrm{CD} 16^{+} /\right.$very late antigen 4 negative) plus a residual population of $\mathrm{CD} 16^{+} /$very late antigen 4 -positive eosinophils. Monocytes were resolved in the mononuclear cell gate based on expression of the LPS receptor CD14. CD14- cells in the mononuclear cell gate were considered lymphocytes. Analysis of leukocyte subsets recovered from blister fluid (Table 2) revealed a significant increase in neutrophils $(P=.014)$, monocytes $(P=.014)$, and eosinophils $(P=.009)$ trafficking into the skin perioperatively in the saline-treated control 
TABLE 1. Perioperative patient characteristics

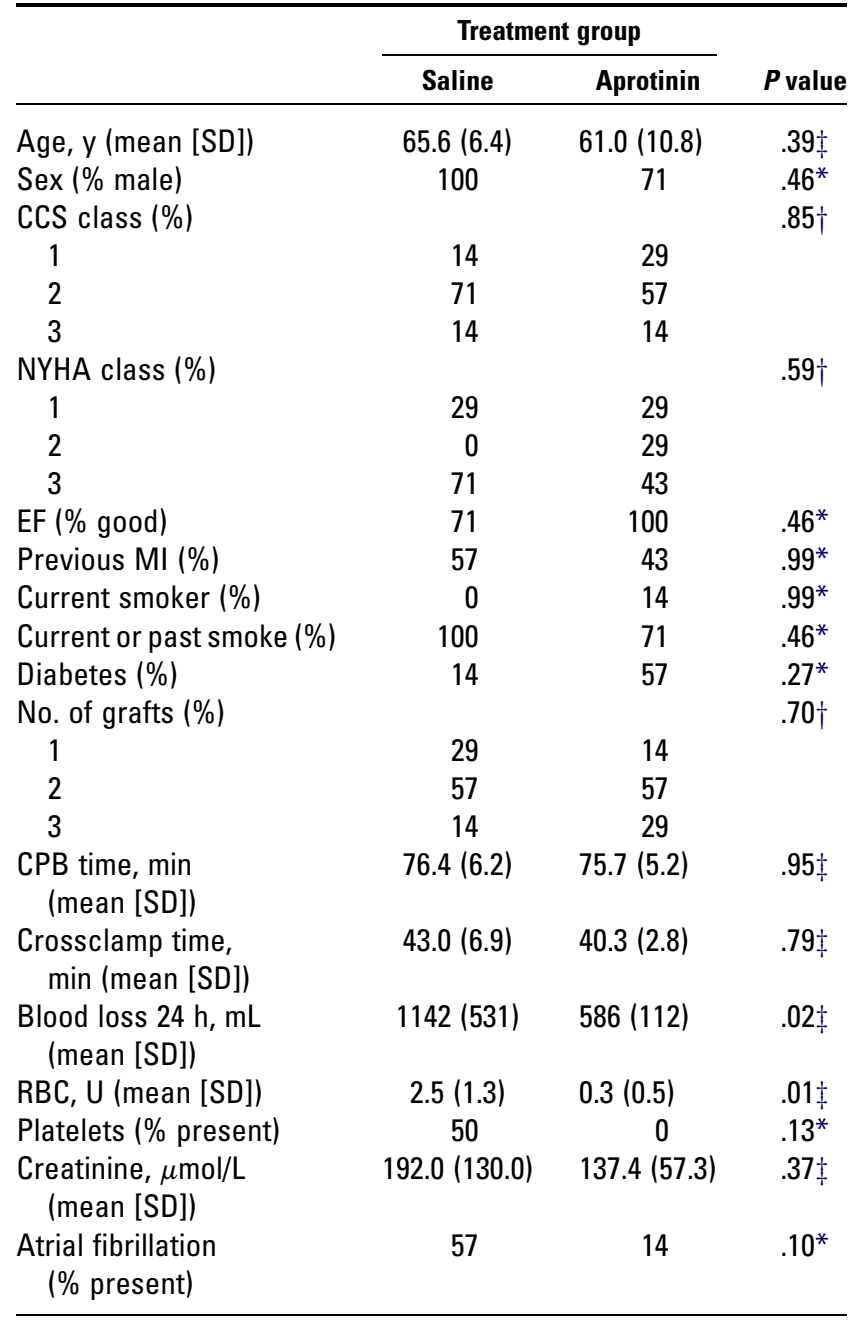

SD, Standard deviation; CCS, Canadian Cardiovascular Society; NYHA, New York Heart Association; $E F$, ejection fraction; $M I$, myocardial infarction; $C P B$, cardiopulmonary bypass; $R B C$, red blood cells. ${ }^{*}$ Fisher exact test. $\dagger$ Wilcoxon Mann-Whitney test for categorical data. $\ddagger$ Permutation test. group of patients undergoing CPB. In contrast, none of these inflammatory leukocyte subsets was statistically significantly increased during surgical intervention in the aprotinin-treated group (Table 2).

\section{Phenotypic Analysis of Emigrated Leukocytes}

The activation phenotype of neutrophils and monocytes infiltrating into blisters was investigated further by comparing CD11b/CD62L expression with the corresponding leukocyte phenotype in blood. Representative flow cytometric histograms (Figure 2, lower panels) depict the upregulation of CD11b (CR3; Mac-1) and shedding of CD62L (L-selectin) receptor on neutrophil and monocyte subpopulations seen during their emigration from blood into blisters. These phenotypic changes, considered a hallmark of leukocyte activation during extravasation, ${ }^{15,23}$ were significant on both neutrophil and monocyte subsets, regardless of treatment group or preoperative versus perioperative time points (Table 3 ). Thus whereas anti-inflammatory intervention with aprotinin prevented operation-induced neutrophil and monocyte trafficking into the skin, the activation status of emigrated leukocytes at 5 hours after the operation was not changed.

\section{Inflammatory Mediators in Blister Fluid}

IL- 8 and TNF- $\alpha$ are examples of inflammatory cytokines that are implicated in organ injury after $\mathrm{CPB} .^{26,27}$ Levels of both cytokines were significantly reduced in perioperative skin blisters in the aprotinin versus saline groups $(P=.044$ for IL-8 and $P=.004$ for TNF- $\alpha$, Figure 3).

\section{Discussion}

The present study establishes the cantharidin skin blister technique as a novel translational tool capable of monitoring the inflammatory effect of cardiothoracic surgery on leukocyte extravasation. By combining the technique with flow cytometric analysis, we have been able to demonstrate for the first time that CPB induces significant extravascular

TABLE 2. Extravasation of leukocytes into skin blisters

\begin{tabular}{|c|c|c|c|c|c|c|c|c|}
\hline & \multicolumn{4}{|c|}{ Saline } & \multicolumn{4}{|c|}{ Aprotinin } \\
\hline & Preoperative & Perioperative & $\begin{array}{c}P \text { value, } \\
t \text { test }\end{array}$ & $\begin{array}{c}P \text { value, } \\
\text { Wilcoxon }\end{array}$ & Preoperative & Perioperative & $\begin{array}{c}P \text { value, } \\
t \text { test }\end{array}$ & $\begin{array}{l}P \text { value, } \\
\text { Wilcoxon }\end{array}$ \\
\hline Total leukocytes & $4.84(2.14-10.92)^{*}$ & $24.48(10.30-58.16)$ & .013 & .043 & $7.52(2.48-22.83)$ & $10.28(4.31-24.49)$ & .470 & .499 \\
\hline Neutrophils & $2.79(1.21-6.44)$ & $14.37(5.64-36.65)$ & .014 & .028 & $4.37(1.41-13.53)$ & $5.18(2.11-12.71) \dagger$ & .724 & .735 \\
\hline Monocytes & $1.16(0.51-2.67)$ & $6.37(2.80-14.49)$ & .014 & .028 & $1.70(0.52-5.53)$ & $2.93(1.13-7.56)$ & .277 & .311 \\
\hline Eosinophils & $0.40(0.18-0.86)$ & $1.73(0.77-3.92)$ & .009 & .018 & $0.76(0.26-2.18)$ & $1.25(0.52-3.01)$ & .266 & .237 \\
\hline Lymphocytes & $0.40(0.16-1.01)$ & $1.02(0.41-2.53)$ & .093 & .063 & $0.58(0.24-1.42)$ & $0.60(0.22-1.59)$ & .972 & .499 \\
\hline
\end{tabular}

${ }^{*}$ Geometric mean (95\% confidence interval); $\times 10^{5}$ cells per blister. †Extravasation of the neutrophil subpopulation into skin perioperatively was marginally inhibited comparing aprotinin versus saline $\left(14.37 \times 10^{5}\right.$ cells per blister after saline and $5.18 \times 10^{5}$ cells per blister after aprotinin: $P=.086$, unpaired $t$ test; $P=.110$, Mann-Whitney test). The same trend was observed for the other leukocyte subsets but was not statistically significant (monocytes: $P=.172$ [ $t$ test], $P=.142$ [Mann-Whitney test]; eosinophils: $P=.568$ [ $t$ test], $P=.565$ [Mann-Whitney test]; and lymphocytes: $P=.357[t$ test], $P=.277$ [Mann-Whitney test]). Overall, the total leukocyte count was marginally inhibited comparing aprotinin versus saline $\left(24.48 \times 10^{5}\right.$ cells per blister after saline and $10.28 \times 10^{5}$ cells per blister after aprotinin: $P=.116$, unpaired $t$ test; $P=.142$, Mann-Whitney test). 
TABLE 3. Fluorescent intensity of CD11b and CD62L on neutrophil and monocyte subsets

\begin{tabular}{llllll}
\hline & \multicolumn{2}{c}{ Saline } & & \multicolumn{2}{c}{ Aprotinin } \\
\cline { 2 - 3 } \cline { 5 - 6 } & Blood MFI & Blister MFI & & Blood MFI & Blister MFI \\
\hline Neutrophil CD11b & & & & & \\
$\quad$ Preop & $1.0(0.9)^{*}$ & $54.0(26.5)$ & & $2.9(2.1)$ & $63.9(37.7)$ \\
$\quad$ Periop & $0.8(0.5)$ & $50.8(28.7)$ & & $1.4(1.0)$ & $54.5(34.1)$ \\
Neutrophil CD62L & & & & & \\
$\quad$ Preop & $97.8(1.5) \dagger$ & $20.6(24.2)$ & $98.9(0.6)$ & $37.4(25.2)$ \\
$\quad$ Periop & $97.5(2.0)$ & $32.7(14.9)$ & $96.8(5.5)$ & $57.1(17.1)$ \\
Monocyte CD11b & & & & & \\
$\quad$ Preop & $0.4(0)$ & $24.3(13.6)$ & & $0.8(0.6)$ & $32.8(22.7)$ \\
$\quad$ Periop & $0.4(0.1)$ & $26.3(18.8)$ & $0.6(0.2)$ & $37.5(26.9)$ \\
Monocyte CD62L & & & & \\
$\quad$ Preop & $61.4(18.0)$ & $2.0(2.8)$ & $73.5(14.3)$ & $13.7(13.8)$ \\
$\quad$ Periop & $74.3(17.0)$ & $1.2(1.0)$ & & $93.5(14.1)$ & $11.4(10.9)$ \\
\end{tabular}

MFI, Mean fluorescent intensity $\left(\log _{10}\right)$. *Mean (standard deviation) CD11b expression was statistically significantly increased on leukocytes recovered from blister fluid compared with values in blood, regardless of leukocyte subtype, preoperative or perioperative time point, or treatment group $(P<.004$, paired $t$ test, in all cases, remaining statistically significant after the Bonferroni correction). $† C D 62 L$ expression was significantly decreased (indicative of shedding) on leukocytes recovered from blister fluid compared with values in blood, regardless of leukocyte subtype, preoperative or perioperative time point, or treatment group $(P<.0001$, paired $t$ test, in all cases, remaining statistically significant after the Bonferroni correction).

migration of each of the major inflammatory leukocyte subsets: neutrophils, monocytes, and eosinophils. Aprotinin attenuated leukocyte extravasation and inflammatory cytokine (IL-8 and TNF- $\alpha$ ) accumulation in blister fluid, thus validating the cantharidin technique as a tool for analyzing antiinflammatory interventions in cardiothoracic surgery.

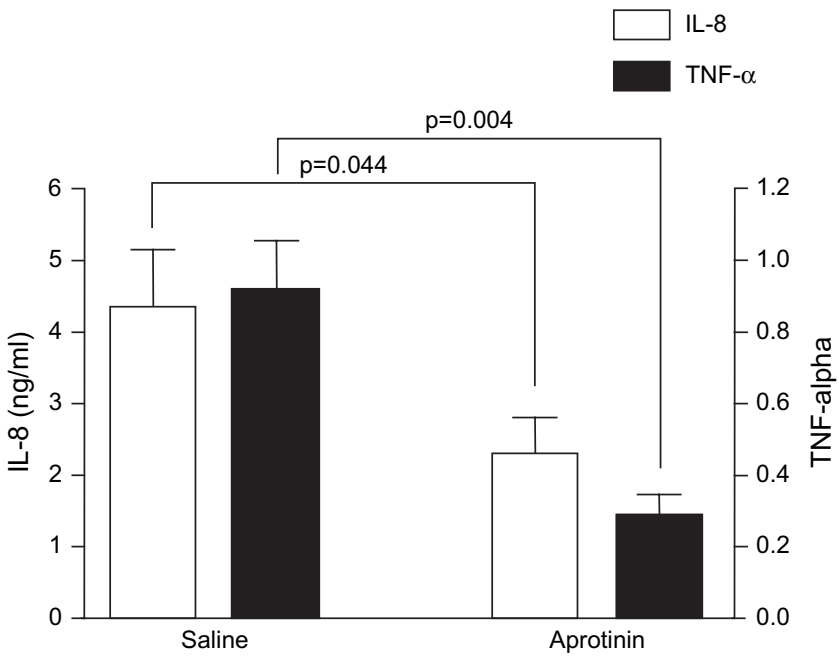

Figure 3. Interleukin (IL) 8 and tumor necrosis factor (TNF) $\alpha$ levels in perioperative blister fluid. TNF- $\alpha$ levels were normalized for each individual relative to preoperative blisters. Results are expressed as the means \pm standard deviation.
Despite the importance of extravascular leukocytes in the pathogenesis of organ injury after CPB, this has been a difficult area to study clinically. Although multiple studies have examined systemic markers of leukocyte activation and systemic inflammatory mediators, these have provided no real insight into the extravascular pool of leukocytes likely to be involved in organ injury. Bronchoalveolar lavage (BAL) fluid is an accessible and clinically relevant extravascular compartment for study but is complicated by the fact that emigrated leukocyte populations can be masked by large resident pools of cells. Studies of BAL fluid have reported accumulation of emigrated neutrophils after $\mathrm{CPB} \cdot{ }^{26,28}$ The present study confirms and extends this work. We demonstrate that neutrophils after surgical intervention (as a percentage) comprise $65.2 \% \pm 19.5 \%$ of total leukocytes in blister fluid. This is greater than previously reported in BAL fluid after surgical intervention $(30.5 \% \pm 12.5 \%$ and $11.3 \% \pm$ $12.2 \%),{ }^{26,28}$ but the smaller neutrophil component in BAL fluid might have been due to the presence of contaminating resident cells in lavage fluid. Our study reinforces the belief that neutrophils play a central role in the pathophysiology of inflammatory organ injury. ${ }^{29}$

Monocytes were the next most abundant tissue-infiltrating cell type $(24.1 \% \pm 6.6 \%$ of total leukocytes in the saline group perioperatively), with the remainder consisting of eosinophils $(6.5 \% \pm 2.0 \%)$ and lymphocytes $(4.2 \% \pm 1.0 \%)$. Monocytes are known to be activated systemically after bypass, ${ }^{30}$ but the observation that these cells emigrate in significant numbers into tissues provides new evidence for a pathogenic role after CPB. Trafficking of activated monocytes, which are a primary source of TNF- $\alpha$, might be of particular relevance to neurologic injury after cardiac surgery because TNF- $\alpha$ causes focal ischemic brain injury through inducing apoptosis. ${ }^{31}$

The present study has also validated cantharidin skin blisters as a tool for monitoring a given anti-inflammatory intervention in cardiothoracic surgery. We present the first in vivo evidence that aprotinin prevents on-pump operation-induced leukocyte extravasation, a mechanism previously imputed from in vitro and animal studies. ${ }^{19-22}$ Inflammatory cytokine (IL-8 and TNF- $\alpha$ ) accumulation was also significantly inhibited by aprotinin. These findings are in agreement with observations in BAL fluid, which reported reduced IL-8 levels in patients receiving aprotinin during CPB surgery. ${ }^{26}$ The combined reduction of inflammatory leukocyte trafficking and proinflammatory cytokine accumulation in skin suggests that these properties of aprotinin might contribute to its neuroprotective mechanism of action demonstrated in placebocontrolled trials. ${ }^{17,18,32}$

The present study was not powered to investigate possible adverse clinical reactions caused by aprotinin administration. ${ }^{33,34}$ Nevertheless, we report no serious adverse events in either treatment group. One patient in the aprotinin group and 2 patients in the saline group had peak serum creatinine 
values of greater than $200 \mu \mathrm{mol} / \mathrm{L}$, but no patient required dialysis. Patients in the aprotinin group had significantly reduced blood loss and red cell transfusion requirement, confirming the hemostatic efficacy of aprotinin. The failure of aprotinin to suppress leukocyte activation in the present study appears at odds with other studies showing inhibition of CD11b by aprotinin on neutrophils examined at earlier time points (15-30 minutes after initiation of bypass). ${ }^{15,23}$ Differences might have been due to the relatively late time point (5 hours postoperatively) of sampling here or the fact that cantharidin skin blisters are intrinsically inflammatory.

The intrinsic inflammatory nature of topical cantharidin is a limitation of the study. What is being sampled postoperatively is a composite of a systemic inflammatory response caused by surgical intervention and a local inflammatory response caused by cantharidin. Nonetheless, surgical intervention with $\mathrm{CPB}$ triggered a robust increase in extravasated leukocytes (381\% compared with reference blisters carried out preoperatively), and this increment was ablated with anti-inflammatory intervention. Because this is the first study to use the cantharidin technique in a surgical setting, it is too early to say whether leukocyte infiltration or cytokine accumulation is correlated with clinical events. Further studies adequately powered to address clinical outcomes would be needed to address this issue. Another limitation is the question of how valid it is to extrapolate anti-inflammatory effects observed in the skin to organs affected by the inflammatory response to $\mathrm{CPB}$. Ideally, one would want a technique for monitoring leukocyte infiltration directly into, for example, the human brain, lung, and kidneys. The value of the cantharidin skin test is that it provides novel molecular insight into the pool of leukocytes migrating extravascularly during the operation, which is poorly understood at present.

In conclusion, we have established a new technique for monitoring the extravascular leukocyte population in cardiothoracic surgery. This technique might be a useful research tool to evaluate future anti-inflammatory interventions in cardiothoracic surgery.

We thank Dr Pete Anning at Scienceposters.co.uk for expert assistance with the figures.

\section{References}

1. Day JRS, Taylor KM. The systemic inflammatory response syndrome and cardiopulmonary bypass. Int J Surg. 2005;3:129-40.

2. Kalman J, Juhasz A, Bogats G, Babik B, Rimanoczy A, Janka Z, et al. Elevated levels of inflammatory biomarkers in the cerebrospinal fluid after coronary artery bypass surgery are predictors of cognitive decline. Neurochem Int. 2006;48:177-80.

3. Ramlawi B, Rudolph JL, Mieno S, Feng J, Boodhwani M, Khabbaz K, et al. C-reactive protein and inflammatory response associated to neurocognitive decline following cardiac surgery. Surgery. 2006;140:221-6.

4. Shann KG, Likosky DS, Murkin JM, Baker RA, Baribeau YR, DeFoe GR, et al. An evidence-based review of the practice of cardiopulmonary bypass in adults: a focus on neurologic injury, glycemic control, hemodilution, and the inflammatory response. $J$ Thorac Cardiovasc Surg. 2006;132:283-90.
5. Clark WM, Madden KP, Rothlein R, Zivin JA. Reduction of central nervous system ischemic injury in rabbits using leukocyte adhesion antibody treatment. Stroke. 1991;22:877-83.

6. Mori E, del Zoppo GJ, Chambers JD, Copeland BR, Arfors KE. Inhibition of polymorphonuclear leukocyte adherence suppresses no-reflow after focal cerebral ischemia in baboons. Stroke. 1992;23:712-8.

7. Day RM, Harbord M, Forbes A, Segal AW. Cantharidin blisters: a technique for investigating leukocyte trafficking and cytokine production at sites of inflammation in humans. J Immunol Methods. 2001;257: 213-20.

8. Honkanen RE. Cantharidin, another natural toxin that inhibits the activity of serine/threonine protein phosphatases types 1 and 2A. FEBS Lett. 1993;330:283-6.

9. Pierard-Franchimont C, Pierard GE. Cantharidin-induced acantholysis. Am J Dermatopathol. 1988;10:419-23.

10. Tromovitch TA. Cantharadin. JAMA. 1971;215:640.

11. Moed L, Shwayder TA, Chang MW. Cantharidin revisited: a blistering defense of an ancient medicine. Arch Dermatol. 2001;137:1357-60.

12. Philippidis P, Mason JC, Evans BJ, Nadra I, Taylor KM, Haskard DO, et al. Hemoglobin scavenger receptor CD163 mediates interleukin-10 release and heme oxygenase-1 synthesis: antiinflammatory monocytemacrophage responses in vitro, in resolving skin blisters in vivo, and after cardiopulmonary bypass surgery. Circ Res. 2004;94:119-26.

13. Evans BJ, McDowall A, Taylor PC, Hogg N, Haskard DO, Landis RC. Shedding of lymphocyte function-associated antigen-1 (LFA-1) in a human inflammatory response. Blood. 2006;107:3593-9.

14. Gott JP, Cooper WA, Schmidt FE Jr, Brown WM III, Wright CE, Merlino JD, et al. Modifying risk for extracorporeal circulation: trial of four antiinflammatory strategies. Ann Thorac Surg. 1998;66:747-53.

15. Hill GE, Alonso A, Spurzem JR, Stammers AH, Robbins RA. Aprotinin and methylprednisolone equally blunt cardiopulmonary bypassinduced inflammation in humans. J Thorac Cardiovasc Surg. 1995; 110:1658-62.

16. Wachtfogel YT, Kucich U, Hack CE, Gluszko P, Niewiarowski S, Colman RW, et al. Aprotinin inhibits the contact, neutrophil, and platelet activation systems during simulated extracorporeal perfusion. J Thorac Cardiovasc Surg. 1993;106:1-9.

17. Buziashvili YI, Ambat'ello SG, Aleksakhina YA, Pashchenkov MV. Influence of cardiopulmonary bypass on the state of cognitive functions in patients with ischemic heart disease. Neurosci Behav Physiol. 2006;36: 107-13.

18. Harmon DC, Ghori KG, Eustace NP, O'Callaghan SJ, O'Donnell AP, Shorten GD. Aprotinin decreases the incidence of cognitive deficit following $\mathrm{CABG}$ and cardiopulmonary bypass: a pilot randomized controlled study. Can J Anaesth. 2004;51:1002-9.

19. Asimakopoulos G, Thompson R, Nourshargh S, Lidington EA, Mason JC, Ratnatunga CP, et al. An anti-inflammatory property of aprotinin detected at the level of leukocyte extravasation. J Thorac Cardiovasc Surg. 2000;120:361-9.

20. Asimakopoulos G, Lidington EA, Mason J, Haskard DO, Taylor KM, Landis RC. Effect of aprotinin on endothelial cell activation. J Thorac Cardiovasc Surg. 2001;122:123-8.

21. Pruefer D, Makowski J, Dahm M, Guth S, Oelert H, Darius H, et al. Aprotinin inhibits leukocyte-endothelial cell interactions after hemorrhage and reperfusion. Ann Thorac Surg. 2003;75:210-5.

22. Anttila V, Hagino I, Iwata Y, Mettler BA, Lidov HG, Zurakowski D, et al. Aprotinin improves cerebral protection: evidence from a survival porcine model. J Thorac Cardiovasc Surg. 2006;132:948-53.

23. Asimakopoulos G, Kohn A, Stefanou DC, Haskard DO, Landis RC, Taylor KM. Leukocyte integrin expression in patients undergoing cardiopulmonary bypass. Ann Thorac Surg. 2000;69:1192-7.

24. Yagnik DR, Evans BJ, Florey O, Mason JC, Landis RC, Haskard DO. Macrophage release of transforming growth factor betal during resolution of monosodium urate monohydrate crystal-induced inflammation. Arthritis Rheum. 2004;50:2273-80.

25. Kirkwood BR, Sterne JAC. Transformations. Essential medical statistics. Oxford: Blackwell Science; 2003. p. 118-28.

26. Hill GE, Pohorecki R, Alonso A, Rennard SI, Robbins RA. Aprotinin reduces interleukin- 8 production and lung neutrophil accumulation after cardiopulmonary bypass. Anesth Analg. 1996;83:696-700. 
27. Nakamura K, Ueno T, Yamamoto H, Iguro Y, Yamada K, Sakata R. Relationship between cerebral injury and inflammatory responses in patients undergoing cardiac surgery with cardiopulmonary bypass. Cytokine. 2005;29:95-104.

28. Alat I, Yuksel M, Buket S, Nalbantgil S, Askar F, Bayindir U, et al. The side-effects of cardiopulmonary bypass on the lungs: changes in bronchoalveolar lavage fluids. Perfusion. 2001;16:121-8.

29. Eppinger MJ, Jones ML, Deeb GM, Bolling SF, Ward PA. Pattern of injury and the role of neutrophils in reperfusion injury of rat lung. J Surg Res. 1995;58:713-8.

30. Rinder CS, Rinder HM, Johnson K, Smith M, Lee DL, Tracey J, et al. Role of $\mathrm{C} 3$ cleavage in monocyte activation during extracorporeal circulation. Circulation. 1999;100:553-8.
31. Barone FC, Arvin B, White RF, Miller A, Webb CL, Willette RN, et al. Tumor necrosis factor-alpha. A mediator of focal ischemic brain injury. Stroke. 1997;28:1233-44.

32. Levy JH, Pifarre R, Schaff HV, Horrow JC, Albus R, Spiess B, et al. A multicenter, double-blind, placebo-controlled trial of aprotinin for reducing blood loss and the requirement for donor-blood transfusion in patients undergoing repeat coronary artery bypass grafting. Circulation. 1995;92:2236-44.

33. Mangano DT, Tudor IC, Dietzel C. The risk associated with aprotinin in cardiac surgery. N Engl J Med. 2006;354:353-65.

34. Mangano DT, Miao Y, Vuylsteke A, Tudor IC, Juneja R, Filipescu D, et al. Mortality associated with aprotinin during 5 years following coronary artery bypass graft surgery. JAMA. 2007;297:471-9.

\section{JTCVS On-Line Manuscript Submission and Review}

The Journal of Thoracic and Cardiovascular Surgery requires authors and reviewers to submit all new and revised manuscripts and reviews via Editorial Manager. Point your browser to http://jtcvs.editorialmanager.com, log in as author or reviewer (as appropriate), and follow the instructions provided.

To retrieve your username and password, click "Forget your password?" on the Editorial Manager log-in page.

If you have questions or experience problems uploading your manuscript or review, please contact the editorial office:

Telephone: 215-762-1854

E-mail: jtcvs@drexelmed.edu 